\title{
Effective of Squats exercises on the variables associated On Leaps performance in exercises
}

\section{"Dr /manal Talat}

\section{Introduction}

Sports has new features reflect the continued progress in all of the associated science and this is what contributes to the development aspects of the physical and technical sports specialist, and that makes us as specialists and researchers in a continuous and constant need to study and experimentation for each new occurs to the sports arena, according to the evolution of many of the sciences led to the development of sports training science.

which has become a distinctive character in the recent period where flourished many sports because of this progress and took the nature of power, speed, precision, agility, and proficiency in the performance of the players the ability kinetic high, so should the workers in the field of sports training and researchers study modern training methods and become acquainted with the results of their application,

so as to ensure the achievement of the best results in short periods of time at the lowest potential .

Squats exercises are considered one of new training methods in which they can develop the performance and development of fitness elements at once.

It has a great benefit in strengthening the muscles of the lower body (thighs and knees, legs and heels) and improves muscle tone.

Where it works to strengthen the muscles of the abdomen and back, which in turn helps to maintain balance while on the move, as it increases the flexibility of the joints (ankles, knees, thighs and lower back), it improves the strength of the muscles of the body and improve job performance. (8: 127-141)(7: 984-998)(8: $1552-1566)(13$ : 428-436)

Mercola 2012 explained that the squats exercises working to

* Exercise Lecturer. Dep.curricula\&Teaching Methods for sports Education Faculty of Sports Education Mansoura University. Assiut Journal For Sport Science Arts 
increase the flexibility of the ankles and hip as they increase the strength of the legs and help increase an individual's ability to jump higher faster.(17)

\section{Exercises Features}

* Squats exercises improve blood circulation and improve the general health of the individual, which contributes to reduce cellulite.

* They are also working to improve the process of digestion.

* You do not need a special place to practice or to certain tools.

* Helps in burning calories leading to weight loss as it works to build muscle.

* Strengthens bones and connective tissue, tendons and ligaments. (18)(16)

"Smith " 2003" and Lutz " 1993 agrees that these exercises is one of the best types of exercises that have the ability to employ multiple muscle groups in one movement. .(11: 629-633). (12: 732-739)

"Essam Abdel-Khalek," 2000, indicates that the performance skills associated capabilities physical motor own closely as mastering the skill performance on the bezel supports the development of the requirements of the performance of special physical and motor capabilities such as muscle power, Coordination, flexibility, balance, and often the skill level of performance will be held by the bezel the acquisition of an individual for this special physical and motor characteristics as seen " AbdelMaksoud," 1997, that training leads to influence the muscles working as it is applied is useful in improving motor performance skills in general and in this regard, "Abdul Aziz el Nemr and" Nariman alKhatib" 1996 adds that the performance improves better if a special type of practitioner training activity and that includes the most important muscles involved in this activity are development and how to use them in the same competition . (4: 165), (2: 411), (1: 189)

Exercises is wider areas and easiest for the exercise of individual activity sports and motor, and because its individually activity practice at any time is something affordable and easy for everyone to do, this beside they develop physical and motor 
characteristics necessary for the individual and the exercise of activities that can not be dispensed with, whether to physically active freestanding or means to prepare the public for various sports activities, a scientifically developed countries reflection in various sports activities in general and in rhythmic exercise in particular. (5:51)

Leaps and jumps of the core body constituent elements of the sentences motor in technical exercise as it is one of the movements that need to be large control body positions with accurate performance in all performance stages and the attendant performance leaps and gaps different conditions in the body, which gives it more beautiful, and requires the performance of those skill availability elements ability and agility, flexibility, balance and neuromuscular compatibility.

Through the researcher experience taught substance exercises college students have noticed a decrease students' performance level in leaps scheduled, which represents the difficulty in performance for students in spite of the effort by the teaching staff, which demonstrated the need for further studies that address training programs attract attention and interaction of the students so went researcher to design squats exercises, which depends on body weight in the first place as it contains exercises variety has its own nature in order to develop special fitness elements leaps which may help to improve the level of per formative them in and firming exercises scheduled to become the output of the best educational process program.

The researcher believes that the problem of this research lies in the low level of fitness and special necessary elements to master leaps in the exercise, along with that setup periods currently require ways and means and methods of training variety to help you master the performance during a specific period of time and it came this research trying to find a style training is a novelty to attract the attention of the students and interact with him even lead the target to be achieved to improve the fitness elements, and had also raise the skill level of performance. 
From this standpoint researcher went to conduct this research to study the effectiveness of Squats exercises on the variables associated on the level of some Leaps in exercises.

\section{Research objectives}

The research aims to identify effectiveness of squats exercises use on:

1- some physical fitness effecting leaps in the exercise elements (star leap, Bend jump, front scissors, back scissors, front leap and horse jump).

2- The level of performance leaps in exercise.

\section{Research hypotheses:}

1- There are significant differences between the two measurements pre and post for the benefit of the dimensional measurement in some fitness elements affecting the leaps in exercises for the experimental group.

2. There are significant differences between the two measurements pre and post for the benefit of the dimensional measurement in performance leaps in the level of exercise for the experimental group

Research procedures: -

First: Research Methodology

The researcher used the experimental method using experimental design with two groups (experimental and control group) in a manner tribal telemetric so as to appropriateness of the nature of the research.

Second: The sample of the research community

The sample from Second Grade Students, Faculty of Physical Education for Girls in Mansoura University for the academic year 2013/2014 and they consists of( 20) students divided into two groups (experimental \& control group).

The experimental group (EG) (10) student trained 3 days a week 35- 55 minutes for unit. The control group (CG) (10) student do the Lecture perform traditional way of teaching while experimental group do Squats exercises to see whether the type of training would have the positive or negative effect on physical fitness effecting leaps in the exercise elements and - the level of performance leaps in exercise.

\section{Training Program}

The 10- week in term consists of (3) lecture per week, total (30) lecture

The Body take a special status during performance and he 
should be maintained during a performance:

- $\quad$ Stand up, feet wide pelvis.

- Contraction of the abdominal muscles.

- $\quad$ Performance as if it were an individual seated on a chair, and the movement is slow.

- Thighs parallel to the floor and stay in this position for several seconds.

- $\quad$ Each exercise leads from 8-12 and repeat visits from twice to three times.

- Rest between sets of 60-90 seconds.(15)(14)

The level of some selected leaps Performance under discussion:

The researcher taking a performance degree level and determine the level of performance of each jump of jumps selected (star jump, arch jump, front scissors, back scissors, front leap and horse

\section{Physical Tests}

30 Second set-up test

* Test lift the trunk of the lie (30s)

* Sergeant vertical jump (cm).

* Back lift

* Leg left jump). with five degrees of measurement tribal and five degrees to measure the post has been taking the average of the total scores each leap alone.

\section{Homogeneity of the sample}

The researcher found homogeneity for the research sample in key variables and the physical and skillful tests under discussion table I.

The Table (I) indicates that the skew coefficients to the sample in basic variables and physical and skill under discussion ranged between -0.12 and 1.5 and these values confined between $( \pm 3)$, which confirms the homogeneity of the sample search in key variables and physical and skill under discussion.

\section{Basic measurements}

Researcher ask experts to choose the component of physical fitness which has relevantly with squats exercises and the result was:

\section{Component}

Abdominal muscular Powe (enclosed 4)

Back muscular power(enclosed 5)

Legs muscular power (enclosed 6)

Back muscular power (enclosed 7)

Legs muscular power 


\section{(enclosed 8)}

* Reverse Sit- ups lie test $(\mathrm{cm})$.

Back flexibility (enclosed 9)

* Grand car test (cm).

Leg flexibility (enclosed 10)

* Skipping rope test (number of

Coordination (enclosed11) times).

* Test stand on the instep (s).

Static balance (enclosed 12)

* Test octagonal (degrees). Dynamic balance (enclosed 13)

\section{Chosen Skillful}

- Star Jump

- Arch Jump

- Front Scissors

- Back Scissors

- Front Leap

- Horse Jump

Table(1)

Homogeneity for the research sample in basic variables and the level for jumps performance

\begin{tabular}{|c|c|c|c|c|c|}
\hline Variables & $\mathbf{U M}$ & Mean & SD \pm & $\begin{array}{c}\text { Media } \\
\text { n }\end{array}$ & Skewness \\
\hline Age & Year & 11.0 &..$\Sigma$ & 11.0 & Zero \\
\hline Weight & $\mathrm{KG}$ & $7 \cdot .74$ & $1 . \wedge \varepsilon$ & $7 . .0$ & 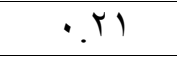 \\
\hline Tall & $\mathrm{CM}$ & 17. & $1 . \wedge \varepsilon$ & 17 & $\cdot . \leqslant \varepsilon$ \\
\hline $\begin{array}{l}\text { Abdominal Muscular } \\
\text { Power }\end{array}$ & $\mathrm{S}$ & $1 \wedge . r V$ & $1 . V T$ & 11.0 &.$T^{T}$ \\
\hline Power Back Muscular & $S$ & $Y \leq .1 T$ & 1.11 & Y纟 &.$r q$ \\
\hline Power Legs Muscular & $\mathrm{CM}$ & TV.TV & 1.20 & tr &.$\leqslant 7$ \\
\hline Back Lift & KG & $V \vee .0$ & $1 . \wedge \varepsilon$ & $V \vee .0$ & Zero \\
\hline Leg Lift & KG & $1 \cdots .7$ & $r .91$ & $1 \ldots$ & .74 \\
\hline Back flexibility & $\mathrm{CM}$ & Tr.T & $1 . \times 1$ & r & $\cdot v \cdot-$ \\
\hline
\end{tabular}

Foolow Table (1)

Homogeneity for the research sample in basic variables and the level for jumps performance 


\begin{tabular}{|c|c|c|c|c|c|}
\hline Variables & UM & Mean & SD \pm & $\underset{n}{\text { Media }}$ & Skewness \\
\hline Age & Year & 11.0 &..$\Sigma$ & 11.0 & Zero \\
\hline Legs flexibility & $\mathrm{CM}$ & $1 V .94$ & 1.20 & 11 & $.1 r_{-}$ \\
\hline Coordination & Number & $1.9 V$ & $\therefore$. Vo & r... & $.1 r_{-}$ \\
\hline Static Balance & $S$ & $r .1$ & $\cdot . \wedge r$ & r.ro & $.0 \varepsilon_{-}$ \\
\hline Dynamic Balance & Degree & $\varepsilon V . r T$ & $0.1 \mathrm{~V}$ & $\sum 7$ & $\because V V$ \\
\hline Star Leap & Degree & r.or & $.0 \leqslant$ & r.o. & .11 \\
\hline Bend Jump & Degree & r.T4 & $.0 \leqslant$ & Y.o. & $\because V T$ \\
\hline Front Scissor & Degree & $r_{.} \varepsilon$. & $\cdot \varepsilon r$ & r.o. & .79 \\
\hline Back Scissor & Degree & Y.Y^ & $\cdot . \wedge \wedge$ & Y.o. & $1 . r v_{-}$ \\
\hline Front Leap & Degree & r.rA & .04 & Y.o & $1 . r \leq-$ \\
\hline Horse Jump & Degree & Y.ro & .0 & $r_{,} \cdot$ & 1.0 \\
\hline
\end{tabular}

$\mathrm{UM}=$ Unit of measurement $\quad \mathrm{SD}=$ standard deviation

$\mathrm{SC}=$ Skewness coefficient

Pre- measurement

The researcher conduct pre- measurement on the research sample in basic, physical and skill variables under study on Sunday 6/10/2013 and Monday $7 / 10 / 2013$.

Implementation of the basic experiment(13)

The researcher ask experts to define the all period of the program(enclosed 3) .

The basic study was conducted in the period from Tuesday 8/10/2013 until Saturday 21/12/2013. for about half two month, 30 lesson,3 lesson per week on Sunday,
Monday and Tuesday of each week, the lesson time from 35 to 55 minutes.

Model of daily unit (enclosed 14)

Post- measurement

After finishing the squats program. Measurement was conduct for the two experimental and control groups on Sunday 22/12/2013 and Monday 23/12/2013 under the same condition.

\section{Statistical treatments:}

After data collection and tabulation. it was Statistically treated by the Statistical program SPSS using the mean, median, standard 
deviation,

Correlation

percentage of improvement.

coefficient, $\mathrm{T}$ - tests and

\section{Results:}

Table (2)

Indication of the differences between the two measurements pre and post and rates of change for the (EG)In physical Variables under consideration $\mathrm{N}=\mathbf{1 0}$

\begin{tabular}{|c|c|c|c|c|c|c|c|c|c|}
\hline \multirow[t]{2}{*}{ Variables } & \multirow[t]{2}{*}{ UM } & \multicolumn{2}{|c|}{$\begin{array}{l}\text { Pre test } \\
\text { average }\end{array}$} & \multicolumn{2}{|c|}{$\begin{array}{l}\text { Post test } \\
\text { average }\end{array}$} & \multicolumn{2}{|c|}{$\begin{array}{c}\text { Difference } \\
\text { between } \\
\text { average }\end{array}$} & \multirow[t]{2}{*}{$\begin{array}{c}\text { T } \\
\text { Value }\end{array}$} & \multirow[t]{2}{*}{$\begin{array}{c}\text { Improvement } \\
\text { Percentage } \\
(\%)\end{array}$} \\
\hline & & $\mathrm{SD} \pm$ & Mean & $\mathrm{SD} \pm$ & Mean & $\mathrm{SD} \pm$ & Mean & & \\
\hline $\begin{array}{c}\text { Abdominal } \\
\text { Muscular } \\
\text { Power }\end{array}$ & Number & $1.7 \leq$ & 19.1 & r. & Y १.६ & 7.1 & $1 \cdot . r$ & rq. & or.q \\
\hline $\begin{array}{c}\text { Back } \\
\text { Muscular } \\
\text { Power }\end{array}$ & Number & $1 . v$ & $r \leqslant .0$ & Y.r & $r v .0$ & 7 & ir & o. & or. 1 \\
\hline $\begin{array}{c}\text { Legs } \\
\text { Muscular } \\
\text { Power } \\
\end{array}$ & $\mathrm{Cm}$ & $1 . \wedge$ & $r V . r$ & Y. & $\varepsilon \cdot r$ & $1 \leq$ & ir & rr.r & $\leq \vee . \wedge$ \\
\hline Back Lift & Kgm & 1.9 & VV.V & 1.9 & $\Lambda ५ . r$ & $\varepsilon . \varepsilon$ & $\Lambda .7$ & rq.1 & 11.1 \\
\hline Leg Lift & Kgm & $r$ & $1 \cdots .7$ & r. & $11 v_{.5}$ & 18.7 & 17.1 & rی.r & 17.8 \\
\hline $\begin{array}{c}\text { Back } \\
\text { flexibility }\end{array}$ & $\mathrm{Cm}$ & $1 . \leqslant 1$ & r & $r . r$ & $\varepsilon 9.7$ & r. & r..r & $\leq r . \wedge$ & 114.9 \\
\hline $\begin{array}{c}\text { Legs } \\
\text { flexibility }\end{array}$ & $\mathrm{Cm}$ & $1 . \wedge \varepsilon$ & IV.r & 1.57 & $7 . v$ & $r \wedge . \varepsilon$ & $1 \cdot .7$ & 11.9 & $\pi . r$ \\
\hline Coordination & Number & $\because V V$ & $r$ &.$v$ & $\leq .0$ & $\varepsilon .0$ & r.o & 11.2 & $1 Y 01$ \\
\hline $\begin{array}{c}\text { Static } \\
\text { Balance }\end{array}$ & $\mathbf{S}$ &..$\leqslant 1$ & $r .00$ & $\because 9$ & $\vee . \wedge$ & 9.11 & $\varepsilon . r$ & ir.r & 119.1 \\
\hline $\begin{array}{c}\text { Dynamic } \\
\text { Balance }\end{array}$ & Degree & 0.1 & $\leq V$ & $\varepsilon . r$ & $\Lambda \leq .0$ & 174.0 & $r v .0$ & $r \wedge$ & $\vee ৭ . \wedge$ \\
\hline Coordination & Number & $\because V V$ & r & $\because V$ & $\varepsilon .0$ & $£ .0$ & Y.o & 11.2 & Iron \\
\hline
\end{tabular}

Table (3)

Indication of the differences between the two measurements pre and post and rates of change for the (EG) In Some leaps in performance level under consideration $N=10$

Assiut Journal For Sport Science Arts 


\begin{tabular}{|c|c|c|c|c|c|c|c|c|c|}
\hline \multirow{2}{*}{$\begin{array}{l}\frac{\tilde{0}}{0} \\
\frac{\pi}{3} \\
\frac{\pi}{3} \\
>\end{array}$} & \multirow{2}{*}{$\sum_{\supset}$} & \multicolumn{2}{|c|}{ 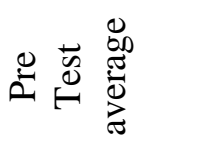 } & \multicolumn{2}{|c|}{ 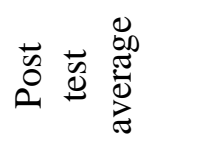 } & \multirow{2}{*}{ 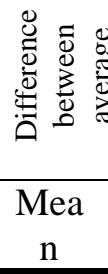 } & \multirow{2}{*}{ 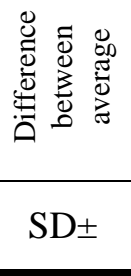 } & \multirow{2}{*}{$H \underset{>}{\stackrel{\mathscr{E}}{ٍ}}$} & \multirow{2}{*}{ 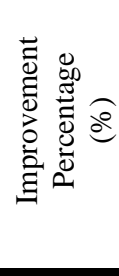 } \\
\hline & & $\mathrm{SD} \pm$ & $\begin{array}{c}\text { Mea } \\
n\end{array}$ & $\mathrm{SD} \pm$ & $\begin{array}{c}\text { Mea } \\
n\end{array}$ & & & & \\
\hline Star Leap & Degree &.$\Upsilon \wedge$ & T.0 & .19 & $\varepsilon .1$ & 1.70 & Y. YO & 11 & 77 \\
\hline Bend Jump & Degree & $\because 0$ & Y. 7 & . rY & $\varepsilon .1$ & 1.7 & Y.E. & 1. & $V Y . V$ \\
\hline $\begin{array}{c}\text { Front } \\
\text { Scissor }\end{array}$ & Degree & $\cdot r$ & Y. 7 &. YO & $\varepsilon . Y$ & 1.70 & $1.0 Y$ & IY.V & 74.0 \\
\hline $\begin{array}{c}\text { Back } \\
\text { Scissor }\end{array}$ & Degree & $\cdot r \wedge$ & Y.o & $\cdot r q$ & $\varepsilon .1$ & 1.70 & r.OY & $\Lambda . Y$ & 77 \\
\hline Front Leap & Degree &.$r \Lambda$ & 1.0 &.$r v$ & $\varepsilon .1$ & 1.7. & $1 . \varepsilon$ & T.r & $7 \varepsilon$ \\
\hline Horse Jump & Degree & .0 & $r . r$ & $\cdot r \cdot$ & $r .9$ & $1 . V$ & r.1. & $11 . r$ & $V \vee . r$ \\
\hline dif & $\begin{array}{cc}\mathrm{T} & \mathrm{va} \\
\text { ole } \mathrm{O}\end{array}$ & & & & $\begin{array}{l}\text { pas } \\
\text { per } \\
\text { lea }\end{array}$ & ve & uren & in & \\
\hline
\end{tabular}

measurement for (EG)\&benefit

\section{Table(4)}

Indication of the differences in dimensional measurement between th(EG) And (CG) in physical attributes under discussion $\mathrm{N}=10$

\begin{tabular}{|c|c|c|c|c|c|c|c|}
\hline \multirow[t]{2}{*}{ Variables } & \multirow[b]{2}{*}{ UM } & \multicolumn{2}{|c|}{ EG)( } & \multicolumn{2}{|c|}{$\overline{\mathrm{CG}})($} & \multirow{2}{*}{$\begin{array}{c}\text { Difference } \\
\text { between } \\
\text { average } \\
\end{array}$} & \multirow{2}{*}{$\begin{array}{c}\mathbf{T} \\
\text { Value }\end{array}$} \\
\hline & & $\mathrm{SD} \pm$ & mean & $\mathrm{SD} \pm$ & Mean & & \\
\hline PAbdominal Muscular Power & Number & r.1 & rq. & $1 . \Sigma 1$ & $1 \wedge . r$ & 11.1 & $I T . Y$ \\
\hline Back Muscular Power & Number & Y.Y & $r v .0$ & Y.o & $r \leq .1$ & $1 \% . \varepsilon$ & $I r . V$ \\
\hline Legs Muscular Power & $\mathrm{Cm}$ & Y.z & $\varepsilon \cdot r$ & r.Yq & $T V_{.0}$ & IY.V & 11.00 \\
\hline Back Lift & Kgm & 1.9 & Aт. & r.Yq & $V V .9$ & ᄉ. & $\wedge . \wedge \varepsilon$ \\
\hline Leg Lift & Kgm & r.IT & $11 V . \varepsilon$ & 0.11 & $1.1 . \mathrm{V}$ & $10 . \mathrm{V}$ & $\vee . \wedge 9$ \\
\hline Back flexibility & $\mathrm{Cm}$ & Y.YE & $\sum 9.7$ & r..0 & Tr.s & YY.Y & Yo.9 \\
\hline Legs flexibility & $\mathrm{Cm}$ & $1 . Y^{4}$ & $7 . \mathrm{V}$ & 1.19 & $I V . V$ & 11 & 10.41 \\
\hline Coordination & Number & $\cdot 7 \mathrm{~V}$ & $\varepsilon .0$ & $.0 r$ & r.l & Y. & $1.0 \mathrm{~V}$ \\
\hline Static Balance & $\mathrm{S}$ &.$\wedge V$ & $\vee . \wedge$ &.$\wedge \Gamma$ & $r .10$ & $\varepsilon .70$ & 11.74 \\
\hline Dynamic Balance & degree & $\leqslant .10$ & $\wedge \varepsilon .0$ & $0 . \cdot r$ & $\leqslant 1.0$ & ru & 17.09 \\
\hline $\begin{array}{l}\mathrm{T} \text { value } \\
\text { the table obs } \\
\text { difference in pa }\end{array}$ & in $(0$ & $=2.1$ & & sica & & attr & \\
\hline
\end{tabular}

Assiut Journal For Sport Science Arts 
Table(5)

Rates of change in the dimensional measurement

between the (EG) And (CG) in the performance leaps level under discussion $\mathrm{N}=10$

\begin{tabular}{|c|c|c|c|c|c|c|c|}
\hline \multirow[t]{2}{*}{ Variables } & \multirow[b]{2}{*}{ UM } & \multicolumn{2}{|c|}{ 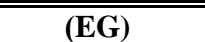 } & \multicolumn{2}{|c|}{$\overline{\text { (CG) }}$} & \multirow{2}{*}{$\begin{array}{c}\text { Difference } \\
\text { between } \\
\text { 5average }\end{array}$} & \multirow[t]{2}{*}{ T Value } \\
\hline & & SD士 & Mean & SD \pm & Mean & & \\
\hline$\overline{\text { Star Leap }}$ & $\begin{array}{l}\text { degree } \\
\end{array}$ & $\overline{0 . r q}$ & $\$ .10$ &. ro & $T \leq 0$ & $1 . \mathrm{V}$ & 1. \\
\hline Bend Jump & degree & 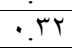 & $\leqslant 10$ & .94 & r.10 & $1 . r$ & $\varepsilon .7$ \\
\hline Front Scissor & degree &.$r_{0}$ & E. YO & . & r.V. & 1.00 & $11.9 r$ \\
\hline Back Scissor & degree &.$r q$ & $\leqslant .10$ &.$Y$ & T.\& & 1.10 & 14.0 \\
\hline Front Leap & degree & $\cdot r V$ & \&.1. & UY & Y.O & 1.7 & $11 . \leqslant T$ \\
\hline Horse Jump & degree & $\cdot r \cdot$ & r.q. & 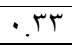 & T.YO & 1.70 & 14.79 \\
\hline
\end{tabular}

$\mathrm{T}$ value in $(0.05)=2.1$ the table observe significant difference in past measurement between (EG)\&(cg)benefit past measurement in the performance of some selected leaps level

Table (6)

Indication of the differences between pre and post measurement and rates of change for the (CG) in the physical attributes under discussion $\mathrm{N}=10$

\begin{tabular}{|c|c|c|c|c|c|c|c|c|c|}
\hline \multirow[t]{2}{*}{ Variables } & \multirow[t]{2}{*}{$\overline{\mathbf{U M}}$} & \multicolumn{2}{|c|}{$\begin{array}{l}\text { Pre test } \\
\text { average }\end{array}$} & \multicolumn{2}{|c|}{$\begin{array}{c}\text { Post } \\
\text { Test } \\
\text { average }\end{array}$} & \multirow{2}{*}{$\begin{array}{c}\begin{array}{c}\text { Difference } \\
\text { Between } \\
\text { average }\end{array} \\
\text { Mean } \\
\end{array}$} & \multirow{2}{*}{$\begin{array}{c}\begin{array}{c}\text { Difference } \\
\text { between } \\
\text { average }\end{array} \\
\text { SD } \pm\end{array}$} & \multirow[t]{2}{*}{$\begin{array}{c}\text { T } \\
\text { Value }\end{array}$} & \multirow[t]{2}{*}{$\begin{array}{c}\text { Improvement } \\
\text { Percentage } \\
(\%)\end{array}$} \\
\hline & & SD \pm & Mean & SD \pm & mean & & & & \\
\hline $\begin{array}{c}\text { Abdominal } \\
\text { Muscular } \\
\text { Power }\end{array}$ & Number & $1.0 \mathrm{~V}$ & 11.1 & $1 . \varepsilon 1$ & $1 \Lambda . r$ & $\because r$ & $9 . \varepsilon$. & זד. & 1.1 \\
\hline $\begin{array}{c}\text { Back } \\
\text { Muscular } \\
\text { Power }\end{array}$ & Number & $1.7 \varepsilon$ & r.q & r.o & $r \leqslant .1$ & $\cdot r$ & 19.7 &.$\leqslant \Gamma$ & $\cdot \wedge \varepsilon$ \\
\hline $\begin{array}{c}\text { Legs } \\
\text { Muscular } \\
\text { Power }\end{array}$ & $\mathrm{Cm}$ & 1.71 & rV.r & r.rq & $r v .0$ & $\cdot r$ & rV. 7 &.$M$ & $\because V r$ \\
\hline Back Lift & Kgm & 1.89 & $V V_{. \varepsilon}$ & Y.rq & VV.q & .0 & 7.0 & $\cdot .71$ & $.7 \varepsilon$ \\
\hline Leg Lift & Kgm & T.AT & $1 \cdots v$ & 0.11 & $1.1 . \mathrm{V}$ & 1 & YYE & .01 & .99 \\
\hline $\begin{array}{c}\text { Back } \\
\text { flexibility }\end{array}$ & $\mathbf{C m}$ & $1.0 \mathrm{~V}$ & rt.l & $r .0$ & rT.乏 & $1 . r$ & or.1 & $1 . \times 1$ & $0 . \wedge \Lambda$ \\
\hline $\begin{array}{c}\text { Legs } \\
\text { flexibility }\end{array}$ & $\mathrm{Cm}$ & I.VA & 11 & 1.89 & IV.V & $\cdot r$ & r^.1 & .00 & 1.77 \\
\hline Coordination & Number & $\cdot v \cdot$ & 1.9 &. or & Y. & $\because r$ & $r .7$ & $1 \ldots$ & $1.0 Y$ \\
\hline $\begin{array}{c}\text { Static } \\
\text { Balance }\end{array}$ & $\mathbf{S}$ &.$\wedge 7$ & $r$ & . & r.10 & .10 & Y. YO & $1 \ldots$ & $0 .$. \\
\hline $\begin{array}{l}\text { the } \\
\text { signifi }\end{array}$ & $\begin{array}{l}\text { T valı } \\
\text { able } \\
\text { ant di }\end{array}$ & $\begin{array}{l}\text { in } \\
\text { obse } \\
\text { eren }\end{array}$ & $\begin{array}{l}05 \\
\mathrm{e} \\
\mathrm{b}\end{array}$ & $\begin{array}{l}=2.26 \\
\text { non- } \\
\text { ween }\end{array}$ & & $\begin{array}{l}\text { past \& } \\
\text { (CG) } \\
\text { attribute }\end{array}$ & $\begin{array}{l}\text { me } \\
\text { cho }\end{array}$ & arem & $\begin{array}{l}\text { at for } \\
\text { aysical }\end{array}$ \\
\hline
\end{tabular}

Table (7)

Assiut Journal For Sport Science Arts 
Indication of the differences between pre and post measurement and rates of change for the (CG) in the performance of some leaps level under discussion $\quad \mathbf{N}=10$

\begin{tabular}{|c|c|c|c|c|c|c|c|c|c|}
\hline \multirow[t]{2}{*}{ Variables } & \multirow{2}{*}{ UM } & \multicolumn{2}{|c|}{$\begin{array}{l}\text { Pre test } \\
\text { average }\end{array}$} & \multicolumn{2}{|c|}{$\begin{array}{c}\text { Post } \\
\text { Test } \\
\text { average }\end{array}$} & \multirow{2}{*}{$\begin{array}{c}\begin{array}{c}\text { Differen } \\
\text { ce } \\
\text { between } \\
\text { average }\end{array} \\
\text { mean } \\
\end{array}$} & \multirow{2}{*}{$\begin{array}{c}\begin{array}{c}\text { Differen } \\
\text { ce } \\
\text { between } \\
\text { average }\end{array} \\
\mathrm{SD} \pm\end{array}$} & \multirow[t]{2}{*}{$\begin{array}{l}\mathrm{T} \\
\mathrm{Va} \\
\text { lue }\end{array}$} & \multirow{2}{*}{$\begin{array}{c}\text { Improve } \\
\text { ment } \\
\text { Percenta } \\
\text { ge } \\
(\%)\end{array}$} \\
\hline & & $\begin{array}{l}\text { SD } \\
\pm\end{array}$ & $\begin{array}{c}\text { mea } \\
\mathrm{n}\end{array}$ & $\begin{array}{l}\text { SD } \\
\pm\end{array}$ & $\begin{array}{c}\text { Mea } \\
\mathrm{n}\end{array}$ & & & & \\
\hline "Star Leap & Degree &. .00 & T.T & .ro & r.£o &. .10 & $1.0 Y 0$ & 11 & 77 \\
\hline $\begin{array}{l}\text { Bend } \\
\text { Jump }\end{array}$ & Degree & .01 & Y. .7 & $.9 r$ & r.10 &. YO & $\begin{array}{c}\text { M.TY } \\
0\end{array}$ & 1. & VY.VO \\
\hline $\begin{array}{l}\text { Front } \\
\text { Scissor }\end{array}$ & Degree & 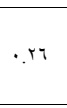 & $r . \leqslant 0$ & $\cdot r r$ & r.v. & . YO & Y.TYO & 15.79 & $7 \pi . \leqslant 7$ \\
\hline $\begin{array}{c}\text { Back } \\
\text { Scissor }\end{array}$ & Degree &.$r r$ & r.ro & $\cdot r$ & r. & $\because .0$ & I.YYO & A.ro & 77 \\
\hline $\begin{array}{l}\text { Front } \\
\text { Leap }\end{array}$ & Degree & $\cdot r r$ & r.ro &.$r Y$ & r. 0 & .10 & $1.0 Y 0$ & $1 r . r T$ & $7 \varepsilon$ \\
\hline $\begin{array}{l}\text { Horse } \\
\text { Jump }\end{array}$ & Degree & $\cdot r Y$ & Y. 1 & $r r$ & r. Yo & .10 & 1.10 & $11 . r r$ & $V V . Y V$ \\
\hline \multicolumn{6}{|c|}{$\begin{array}{l}\text { T value in }(0.05)=2.26 \\
\text { the table observe non- } \\
\text { significant difference between } \\
\text { pre \& past measurement for } \\
\text { (cg) in the performance of } \\
\text { some selected leaps level. } \\
\text { Discussion } \\
\text { Evident from the table } \\
\text { (2) the existence of statistically } \\
\text { significant differences between } \\
\text { pre and post measurement for } \\
\text { the telemetric experimental } \\
\text { group in the physical attributes } \\
\text { selected under discussion. } \\
\text { Attributed this statistical } \\
\text { significance to the regular } \\
\text { training for squats exercises } \\
\text { that led to the rise of fitness for }\end{array}$} & \multicolumn{4}{|c|}{$\begin{array}{l}\text { the students level was the } \\
\text { highest ratios of Coordination } \\
\text { rate by percentage (125.00\%), } \\
\text { followed by a Static balance } \\
\text { component by percentage } \\
(119.72 \%) \text { then the spine } \\
\text { flexible rate by percentage } \\
(112.88 \%) \text { and the last of the } \\
\text { power element back muscles } \\
\text { by percentage (11.07\%) and } \\
\text { the researcher attributed these } \\
\text { differences to the proposed } \\
\text { program as the regularity in the } \\
\text { exercise program led to } \\
\text { positive changes and the } \\
\text { effects in terms of physical and } \\
\text { this is consistent with what } \\
\text { indicated by each of Escamilla }\end{array}$} \\
\hline
\end{tabular}


2001, McCaw, ST and Melrose1999, Escamilla RF, Fleisig, GS, Lowry2001. (9: 127-141)(7:984-998)(8: 15521566)(13:428-436)

Where he explained that the squats exercises have the ability to develop performance and fitness elements at the same time to develop.

It has a great benefit in strengthening the muscles of the lower part of the body and improves muscle tone. And squats exercises work to strengthen the muscles of the abdomen and back, which in turn helps to maintain balance while on the move, as it increases the flexibility of the joints (ankles, knees, thighs and lower back) and improved power muscles of the body and improve job performance. And this may have been validated on the first hypothesis, which states that: There are significant differences between the two measurements pre and post for post-measurement in some fitness elements affecting the leaps in exercises for the experimental group.

Evident from the table (3) and no statistically significant differences between the experimental group and the control group in the posttest performance measurement in selected leaps level under discussion for the experimental group.

Researcher Due these differences to the proposed program as the regularity in the exercise followed the program three times a week for 10 weeks, which contains a variety of exercises for all parts of the body and contains a coherent set of leaps in the exercise scheduled on the second band, where they raise their motivation to compete with each other and try to good whom tradition, which is trying to show its superiority over the rest of the students has led to changes and positive effects in terms of skill for the students and that as a result of the positive changes that have occurred in terms of physical as physically and skill influenced, each other and were the highest proportion of horse jump by (77.27\%), followed by arched jump by (72.75) and most recently by the front leap $(64 \%)$.

This is consistent with Essam Abdel-Khalek, "2000," where he pointed out that the performance skills associated capabilities physical kinetic 
own closely where mastering the skill performance on the bezel supports the development of the requirements of the performance of the physical capabilities and mobility, especially as the skill level of performance will be held by the extent of the acquisition of the individual to these qualities physical and kinetic own also agree with "Mr. AbdelMaksoud," 1997 ", which indicated that the $\mathrm{m}$ training leads to influence the muscles working as it is applied is useful in improving motor performance skills in general. (4: 165), (2: 411), (1: 189).

This may be achieved on the second hypothesis, which states that: There are significant differences between the

two

measurements pre and post for post-measurement leaps in performance level in the exercises for the experimental group.

It is clear from Table (6) and the existence of differences between the measurement pre and post, but not statistically significant for the control group in the physical attributes and the highest percentage of component compatibility and were accounted for $(10.52 \%)$, followed by the spine flexiblity by $(5.88 \%)$ The lowest percentage of the strength back muscles by $(0.64 \%)$.

As can be seen from the table (7) and the existence of differences between the measurement pre and post, but not statistically significant for the control group in the performance of these selected leaps level and is also evident from the same table, and having a simple improvement between the two measurements pre and post for the benefit of the dimensional measurement in all selected leaps ratios were front scissors jump (10.20\%) is the highest percentage of improvement followed by Arch jump $(9.61 \%)$ and the last of them back scissors jump that percentage $(2.12 \%)$, and this improvement is a result of the program adopted in college.

\section{Conclusions:}

Within the limits of the search procedures in the light of the objectives and analysis of the results reached the researcher to the following conclusions:

* The proposed program of squats exercises a positive impact on the physical variables (under discussion) a positive impact. 
* The proposed program of squats exercises a positive impact on the leaps that have been taught to students a positive impact and in favor of the experimental group.

* Rates of change of the experimental group have higher than the control group in all search physical and skill variables.

\section{Recommendations:}

Within the limits of the results of the research sample and the researcher recommends the following:

* The use of the proposed program to squats exercises for the students to different college teams in different sports activities because of its positive effects on all the variables under consideration.

* Use of the proposed program for the squats exercises in raising the performance of different types of skills in the exercise.

* Further scientific studies and similar research study on the different stages of the sexes in college to improve the physical aspect and skill to have.

\section{References:}

Arabic References

1. Alsaid abd el maqsoad: Theorems of physical training (Power physiology training), cairo ,Book center publish, 1997.

2. Abd el aziz el nemr, Nariman el Khateab: Weights training, Designing Power program \& planning Athletic Season, Cairo, cairo center publish, 1996.

3. Essam Abd ElKhalek: Athlete Training, Theorems \& Implementations, E 10, Alex, Dar el maref 2000.

4. Enayat Ali Labib, Briksan Othman Hussain: Exercises \& Rhythmic Gymnastic, Physical education gzira, Helwan University, 2001.

\section{Laila}

Zahran:

Academic \& Practicability Fundamentals for exercises \& Rhythmic Gymnastic, Dar el Fekr el Araby, Cairo, 1997.

\section{English References}

7- Donnelly, David V ; BERG, William P; Fiske, Darryn M. Journal of Strength \& Conditioning Research: February 2006.

8- Escamilla, RF, Fleisig, GS, Lowry, TM, Barrentine, SW, and Andrews, JR. A threedimensional biomechanical analysis of the squat during varying stance widths. Med Sci Sports Exerc 33: 984-998, $2001 b$.

9- Escamilla, RF, Fleisig, GS, Zheng, N, Lander, JE, 
Barrentine, SW, Andrews, JR, Bergemann, BW, and Moorman, CT. Effects of Technique variations on knee biomechanics during the squat and leg Press. Med Sci Sports Exerc 33: 1552-1566, 2001a. 10- Escamilla, RF. Knee biomechanics of the dynamic squat exercise. Med Sci Sports Exerc 33: 127-141, 2001.

11- Esformes, Joseph2013 Journal of Strength \& Conditioning Research: November 2013 - Volume 27 Issue 11 - p 2997-3000 doi: 10.1519/JSC.0b013e31828d44 65

12- Fry, AC, Smith, JC, and Schilling, BK. Effect of knee position on hip and knee torques during the barbell squat. J Strength Cond Res 17: 629-633, 2003.

13- Lutz, GE, Palmitier, RA,
An, KN, and Chao, EY. Comparison of tibiofemoral joint forces during open kinetic chain and closed kinetic chain exercises. J Bone Joint Surg Am 75: 732-739, 1993.

14- McCaw, ST and Melrose, DR. Stance width and bar load effects on leg muscle activity during the parallel squat. Med Sci Sports Exerc 31: 428-436, 1999.

15- Whyprrelink "http://www. beautyandtips.comsport/"ww.b eautyandtips.com-sport and fitness.

16- www. Fitday.com -fitnessarticle.

17- www. Liverstrong.comarticle403187-what are the benifite of doing a basic squat 18- www.drmeracola.com/ exercise

19- www.nikkigunz. comfittness-tips. 